\title{
Speech impairment in a large sample of patients with Parkinson's disease
}

\author{
Aileen K. $\mathrm{Ho}^{\mathrm{a}, *}$, Robert Iansek ${ }^{\mathrm{b}}$, Caterina \\ Marigliani $^{\mathrm{b}}$, John L. Bradshaw ${ }^{\mathrm{a}}$ and Sandra \\ Gates $^{b}$ \\ ${ }^{a}$ Neuropsychology Research Unit, Psychology \\ Department, Monash University, Clayton 3168, \\ Victoria, Australia \\ ${ }^{\mathrm{b}}$ Kingston Centre, Warrigal Road, Cheltenham 3192, \\ Victoria, Australia
}

\begin{abstract}
This study classified speech impairment in 200 patients with Parkinson's disease (PD) into five levels of overall severity and described the corresponding type (voice, articulation, fluency) and extent (rated on a five-point scale) of impairment for each level. From two-minute conversational speech samples, parameters of voice, fluency and articulation were assessed by two trained-raters. Voice was found to be the leading deficit, most frequently affected and impaired to a greater extent than other features in the initial stages. Articulatory and fluency deficits manifested later, articulatory impairment matching voice impairment in frequency and extent at the 'Severe' stage. At the final stage of 'Profound' impairment, articulation was the most frequently impaired feature at the lowest level of performance. This study illustrates the prominence of voice and articulatory speech motor control deficits, and draws parallels with deficits of motor set and motor set instability in skeletal controls of gait and handwriting.
\end{abstract}

Keywords: Parkinson's disease, speech impairment

\section{Introduction}

Though often overshadowed by the more salient and debilitating skeletal aspects of movement impairment such as gait and upper limb control, speech impairment in Parkinson's disease (PD) is not uncommon. The existing body of acoustic and kinematic studies of patients with PD typically involve small samples, and findings have been somewhat inconsistent. This has been attributed to the variability of the presenting speech impairment in patients with PD [8], and has been a limitation rather than the main focus of research.

${ }^{*}$ Corresponding author: Aileen K. Ho, Department of Psychology, Monash University, Clayton 3168, Victoria, Australia. Tel.: +61 39755 5616; Fax: +61 39905 3948; E-mail: Aileen.Ho@sci.monash.edu.au.
Only two large-scale speech studies of patients with PD have been conducted [3, 6], estimating the prevalence of speech deficit to be approximately $49 \%$ and $70 \%$ respectively. Logeman et al's [6] clinicalperceptual study examined the frequency and cooccurrence of voice, articulation and fluency impairment in 200 PD patients. They reported that $89 \%$ experienced voice disorders, $45 \%$ experienced articulatory impairment and $20 \%$ experienced problems with fluency. By examining the co-occurrence of speech deficit, a progression of impairment was hypothesized; beginning with voice, followed by articulation. It progressed from posterior lingual involvement eventually (and most anteriorly) to labial involvement.

Whilst inherently subjective, the use of clinicalperceptual ratings as in [6] is uniquely suited to examining aspects of patients' speech impairment that are most relevant from the perspective of their functional communication needs [1]. For this purpose, clinical-perceptual judgements are ecologically valid and indeed more appropriate than acoustic and kinematic methods, which may not be readily translated to patients' everyday ability to speak in a communicative setting.

This study uses the clinical-perceptual method to explore further the nature of Parkinsonian speech difficulties by examining the speech deficit profiles (voice, articulation and fluency) of a cross-section of speech impaired patients with PD. We sampled the speech of 200 patients and classified their overall speech function into five categories of severity. However, instead of merely noting the absence or presence of voice, articulation and fluency deficits, as in [6], the extent of impairment was rated. Hence, the prevalence of qualitative measures, i.e., voice, articulation and fluency deficits, could be examined across levels of overall speech impairment, as also could quantitative measures, i.e., the degree to which these specific parameters of speech are impaired. The simultaneous investigation of the qualitative and quantitative aspects of Parkinsonian speech in a large sample provides epidemiological information regarding the prevalence of levels of general speech impairment, and the specific 
parameters of speech motor control. It also permitted a detailed description of the common profiles of deficit to provide further insight into the clinical course of Parkinsonian speech impairment.

\section{Method}

\subsection{Participants}

Two hundred successively-presenting idiopathic PD patients (77 females, 123 males; mean age $=70.8$ years, standard deviation $=9.1$ years) from the Kingston Centre Movement Disorders Clinic participated in this study. Diagnosis of idiopathic Parkinson's disease was made by a neurologist (RI), utilising the criteria of Hughes, Daniel, Blankson and Lees [4]. Patients ranged from mild to severe on the Webster scale of PD symptom severity [17], and were all on anti-Parkinsonian medication. The time of speech sampling with respect to medication phase was kept random, occurring whenever patients arrived at the clinic. Patients with dyskinesia, however, were excluded. Ethical approval was obtained and all participants gave informed consent for this study.

\subsection{Apparatus and procedure}

Two-minute conversational speech samples were taped using a Marantz portable cassette recorder (PMD222) in quiet examination rooms during regular outpatient visits. Patients were asked to talk about familiar conversational topics such as family and recreational activities.

The speech samples were rated by two trained raters (interater reliability was 0.90 , intrarater reliability was 0.95) using Part A (motor deficit profile) of the (FPCP) Functional Parkinson's Communication Profile ${ }^{1}$ [7]. This communication profile (see Appendix 4) assesses three features of speech motor control, i.e., voice (e.g., harsh quality, reduced volume, disturbed intonation), fluency (e.g., motor initiation difficulties, inappropriate pauses, syllable repetition, rushes of speech) and articulation (undershooting of articulatory movement resulting in imprecise articulation). These speech features were rated on a five-point scoring system, where 5 (Level 5) corresponds to $80-100 \%$ functional production, 4 (Level 4) to 60-80\%, 3 (Level 3) to 40

\footnotetext{
${ }^{1}$ Full information on this profile is available from the authors upon request.
}

$60 \%, 2$ (Level 2) to 20-40\% and 1 (Level 1) to 0$20 \%$. The details of the procedure are outlined in Appendix 4. The motor speech profile was scored according to the standard Guttman cumulative scaling system [15] which increases the sensitivity of the instrument by magnifying the range of scores. Each feature received the score for the rated level of functioning as well as all lower levels. For example, if a patient's voice was rated at Level 4 , his score for voice would be $10(4+3+2+1)$. Therefore the highest total (overall) score possible was 45 (voice, fluency and articulation all at Level 5, i.e., $15+15+15)$ and the lowest 3 (voice, fluency and articulation all at Level 1, i.e., $1+1+1)$.

\section{Results}

Firstly, the overall speech function of participants was classified into categories of severity. This was based on predominant voice impairment as the latter is likely to reflect the global progressive deterioration of speech in PD, see [6]. At each score along the spectrum of total speech scores found in this sample, the percentage of predominant voice impairment (i.e., cases where voice was the most affected feature, relative to fluency and articulation) was noted, see Table 1. Predominant voice impairment was sensitive to overall speech impairment across the spectrum of total scores, occurring very frequently with mild overall speech impairment and gradually less frequently in more severe cases. Therefore, this procedure was used to divide the scoring continuum into emerging levels of severity, rather than by applying arbitrary cut-off scores. Five clear classificatory groups of total speech scores emerged from this continuum. Of the sample of 200 patients, $26.0 \%$ were not impaired, $18.0 \%$ were Mildly impaired, $34.0 \%$ Moderately, $18.5 \%$ Severely and $3.5 \%$ Profoundly.

Fig. 1 examined the frequency of impairment of voice, fluency and articulation within the categories of overall speech severity. Generally, the occurrence of voice impairment was very frequent across all categories of severity. However, fluency and articulatory impairment gradually increased in frequency as speech was more severely affected.

To explore speech impairment in greater detail, the data were plotted in three dimensions to reveal further quantitative information on the degree of impairment (note that Level 1 = lowest level of performance), of voice, fluency and articulation within the categories of overall speech severity, see Fig. 2. 
Table 1

Mean percentage of predominantly voice impaired cases for different severity levels of speech impairment

\begin{tabular}{|c|c|c|c|c|}
\hline $\begin{array}{l}\text { Total speech } \\
\text { scores in } \\
\text { ascending order }\end{array}$ & $\begin{array}{l}\text { Proportion of } \\
\text { predominantly voice } \\
\text { impaired cases }\end{array}$ & $\begin{array}{l}\% \text { of predominantly } \\
\text { voice impaired } \\
\text { cases }\end{array}$ & $\begin{array}{l}\text { Mean } \% \text { of } \\
\text { voice impaired } \\
\text { cases }\end{array}$ & $\begin{array}{l}\text { Category } \\
\text { of severity }\end{array}$ \\
\hline 45 & $0 / 0$ & 0.0 & 0.0 & not impaired \\
\hline 40 & $34 / 36$ & 94.4 & 94.4 & mild \\
\hline 36 & $11 / 16$ & 68.8 & \multirow{7}{*}{71.9} & \multirow{7}{*}{ moderate } \\
\hline 35 & $8 / 12$ & 66.7 & & \\
\hline 33 & $5 / 6$ & 83.3 & & \\
\hline 31 & $14 / 21$ & 66.7 & & \\
\hline 30 & $0 / 5$ & 0.0 & & \\
\hline 28 & $3 / 4$ & 75.0 & & \\
\hline 27 & $0 / 3$ & 0.0 & & \\
\hline 26 & $7 / 12$ & 58.1 & \multirow{6}{*}{46.0} & \multirow{6}{*}{ severe } \\
\hline 24 & $4 / 7$ & 57.1 & & \\
\hline 23 & $2 / 2$ & 100.0 & & \\
\hline 22 & $2 / 9$ & 22.2 & & \\
\hline 21 & $0 / 2$ & 0.0 & & \\
\hline 19 & $2 / 5$ & 40.0 & & \\
\hline 18 & $0 / 1$ & 0.0 & \multirow{4}{*}{14.3} & \multirow{4}{*}{ profound } \\
\hline 15 & $1 / 1$ & 100.0 & & \\
\hline 12 & $0 / 3$ & 0.0 & & \\
\hline 9 & $0 / 2$ & 0.0 & & \\
\hline
\end{tabular}

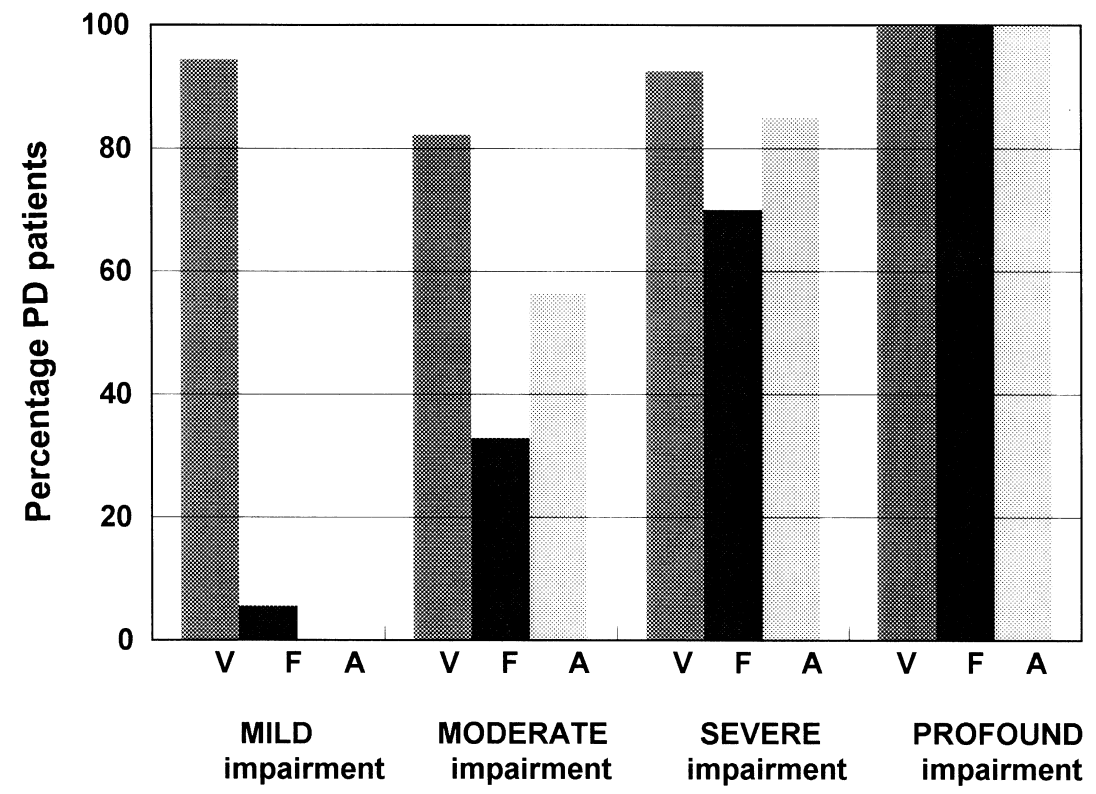

Fig. 1. Percentage of voice (V), fluency (F) and articulatory (A) impairment for each category of severity.

The frequency of voice impairment in the Mild (Fig. 2a) and Moderate (Fig. 2b) stages, shown earlier, is further emphasised by its relative severity. For the Severe stage (Fig. 2c), voice and articulation were the most severely affected parameters. For the Profoundly impaired stage (Fig. 2d), articulation was impaired to the greatest extent, even though all three parameters were equally frequently impaired (as shown earlier in Fig. 1).

From this cross-sectional analysis of various severity levels of Parkinsonian speech dysfunction, voice deficit was found to be the leading symptom, the most frequently affected, and impaired to a greater extent than other features in the initial stages. Articulatory 


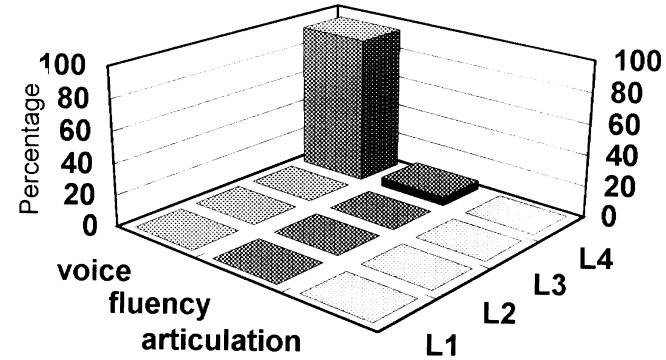

a) MILD IMPAIRMENT

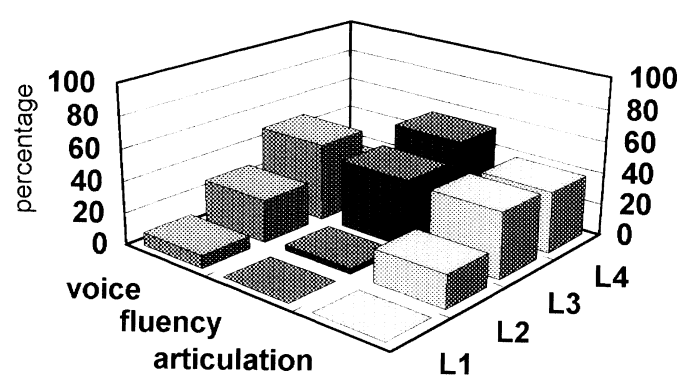

c) SEVERE IMPAIRMENT

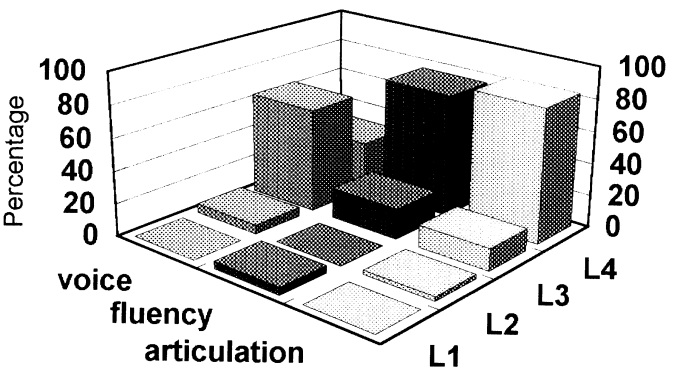

b) MODERATE IMPAIRMENT

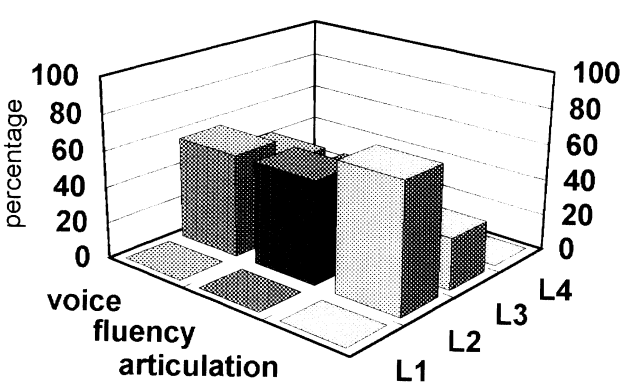

d) PROFOUND IMPAIRMENT

Fig. 2. Percentage of voice, fluency and articulatory impairment according to levels of functional impairment (Level 1 most impaired) for each category of severity.

and fluency deficits manifested later; articulatory impairment matched voice impairment in frequency and extent when at the Severe stage, before becoming the most frequently impaired feature, and to the greatest extent, at the Profound stage.

Patients' speech profiles were also examined for patterns of co-occurrence in terms of presence of impairment, regardless of extent. Three particularly prominent patterns of impairment emerged: firstly, voice impairment only (25\%), secondly, voice impairment with additional articulatory deficit (18.5\%), and thirdly, voice impairment with additional articulatory and fluency deficits (i.e., global impairment, 15.5\%). In general, the presence of voice impairment was highest $(65.5 \%)$ in this sample, followed by articulatory impairment (38.5\%) and finally, fluency (29.0\%).

\section{Discussion}

This study classified speech impairment in 200 patients with PD into five levels of overall severity and described the corresponding type (voice, articulation, fluency) and extent (Level 1 to Level 5) of impairment. Approximately a quarter of patients (26.5\%) showed no speech impairment. The remaining participants $(73.5 \%)$ demonstrated a gradual deterioration of speech features, almost always involving voice first, before progressing to the prominent voice and articulation pattern, with the latter being the most severely affected relative to voice and fluency impairment at the Profound stage. We found a minority of cases of pure articulation (1\%) and fluency impairment (3.5\%) alluded to by [6]. While this illustrates that fluency and articulatory deficits may, albeit rarely, exist in isolation, it confirms the dominance of voice impairment. Voice dysfunction, initially marked by a change in quality, later led to a reduction in speech volume, especially dramatic in more severe cases.

The overwhelming prevalence of voice disturbance and volume reduction is similar to other common Parkinsonian phenomena such as reduced stroke length in handwriting [14] and reduced stride length [9]. The amplitudes of responding in complex motor skills such as speech, stroke length (upper limb) and stride length (lower limb gait) are scaled automatically prior to the execution of the motor plan. In PD, amplitude appears to be pre-set at a lower-than-normal level. This miniaturisation of the motor plan is consistent with the 
hypothesis of reduced cortical motor set due to basal ganglia (BG) dysfunction [5]. Akinetic difficulties in gait associated with hesitation, festination and motor block can be compared to fluency difficulties seen in PD speech. Fluency deficits $(29 \%)$ were markedly less common than reduced volume $(65.5 \%)$, mirroring the less frequent akinetic symptoms (halting gait $25 \%$, festination $4 \%$ according to [14] relative to the typical stride length disturbance in gait $(78 \%$ according to [11], though both akinetic symptoms, and fluency as we have here found, can also occur in isolation.

Another probable function of the BG is a role in the provision of internal cues to enable the smooth, sequential execution of sub-movements within a motor plan [5]. Defective internal BG cuing in PD has been suggested to result in progressive decrements in amplitude over the duration of the motor sequence, a phenomenon known also as motor instability [5]. This cue deficit is seen in the gradual reduction of movement amplitude in gait and hand-writing, as each successive stride length and stroke length becomes progressively smaller and smaller. Articulation impairment could be compared to the lower and upper limb demonstration of cue deficit over a sequence of sub-movements and could likewise be attributed to defective internal BG cueing in PD. In addition to that, the two phenomena, the small amplitude due to motor set disturbance in handwriting and gait, and the motor instability due to insufficient cueing, are usually superimposed, as in our present study where voice and articulation difficulties commonly co-occurred.

Despite the different mechanical properties, parallels between the complex motor skills of speech and limb control have been suggested (e.g., $[13,16]$ ). Other studies (e.g., $[12,2]$ ) that have found contrary evidence to this have attributed the difference to the vastly dissimilar biomechanical properties, but do not necessarily preclude the possibility of underlying commonalities in higher order neural control. Indeed the organisation of meaningful speech output (as opposed to artificial syllable repetition) is likely to resemble other meaningful and intentional limb-related motor behaviours such as handwriting and ambulation.

From the pattern of prevalence seen in the parameters of speech impairment, this study suggests that the pathophysiological perspective on speech disturbance in PD may be comparable to that which relates to limb movement deficits. It also provides a useful clinical classification, as attempts (which have proved successful in limb movements [10]) at improving problems both of motor set and cue may also prove helpful in speech rehabilitation; such a perspective therefore forms a valuable framework for clinical management of speech problems in PD.

\section{Appendix A: Kingston centre profile for functional communication in Parkinson's disease}

Part A: Motor deficit profile

5 points (80-100\% functional)

VOICE

FLUENCY

ARTICULATION

4 points (60-80\% functional)

VOICE

FLUENCY

ARTICULATION

3 points (40-60\% functional)

VOICE

FLUENCY

ARTICULATION

2 points (20-40\% functional)

VOICE

FLUENCY

ARTICULATION

1 point (0-20\% functional)

VOICE

FLUENCY

ARTICULATION

TOTAL SCORE:

\section{Appendix B: Functional Parkinson's communication profile (FPCP) guidelines}

Voice

RATING DESCRIPTION: 5(b)

Volume is adequate in any conversational setting. Able to adjust volume according to environmental needs.

Voice quality is within normal limits (Oates, 1981). 
Intonation is appropriate to conversational context.

\section{RATING DESCRIPTION: 4(b)}

Volume is adequate in both controlled/quiet and everyday environments.

$\square$ Mild difficulty in adjusting volume in response to environmental changes.

Mild harshness, breathiness and/or resonance changes may be preset.

Mild intonation changes may be present.

\section{RATING DESCRIPTION: 3(b)}

$\square$ Moderate difficulty adjusting volume in response to environmental change.

Moderate harshness, breathiness and/or resonance changes may be present.

Moderate intonation changes may be present.

\section{RATING DESCRIPTION: 2(b)}

Volume is adequate in controlled and quiet $1: 1$ environments $20-40 \%$ of the time.

$\square$ Severe difficulty in adjusting volume according to environmental demands.

Severe changes to voice quality, with occasional aphonia.

$\square$ Monotonous voice.

\section{RATING DESCRIPTION: 1(b)}

Volume is non-functional, even in controlled and quiet $1: 1$ environments.

$\square$ Unable to adjust volume according to environmental demands.

\section{Articulation}

\section{RATING DESCRIPTION: 5(c)}

Speech is intelligible in all conversational situations.

$\square$ Articulatory gestures are complete.

Speech rate is within normal limits.

\section{RATING DESCRIPTION: 4(c)}

Speech is intelligible in $60-80 \%$ of utterances.

Speech in intelligible in all controlled settings. Mildly compromised intelligibility in other environments.

Mild articulatory undershooting.
Speech rate is mildly affected.

RATING DESCRIPTION: 3(c)

Speech is intelligible in $40-60 \%$ of utterances.

$\square$ Speech is intelligible in controlled settings however increased attention is required by the listener.

$\square$ Moderate articulatory undershooting.

Speech rate is moderately affected.

RATING DESCRIPTION: 2(c)

$\square$ Speech is intelligible in $20-40 \%$ of utterances.

$\square$ Speech is inconsistently intelligible in controlled settings and maximal attention is required of the listener.

Severe articulatory undershooting.

Speech rate is severely increased or decreased.

\section{RATING DESCRIPTION: 1(c)}

$\square \quad$ Speech is rarely intelligible to unfamiliar listeners. Occasionally intelligible to carers.

Fluency

RATING DESCRIPTION: 5(d)

Rate and rhythm of speech are intact.

Able to initiate, sequence and end speech without difficulty.

RATING DESCRIPTION: 4(d)

Speech is mildly dysfluent.

Evidence of rapid bursts of speech.

Co-ordination of respiration and speech is mildly affected.

$\square$ Evidence of mild initiation and/or sequencing problems.

RATING DESCRIPTION: 3(d)

Speech is moderately dysfluent in $40-60 \%$ of utterances.

$\square \quad$ Frequent bursts of rapid speech.

$\square$ Moderate problems in initiating, sequencing and/ or ceasing articulation.

$\square$ Evidence of blocking and/or phoneme/syllable repetition in $40-60 \%$ of utterances.

RATING DESCRIPTION: 2(d)

Speech is severely dysfluent. 
Frequent bursts of rapid speech.

Co-ordination of respiration and speech is severely affected.

Severe problems in initiating, sequencing and ceasing articulation.

Evidence of frequent blocking, phoneme/syllable repetition.

RATING DESCRIPTION: 1(d)

Speech is non-fluent.

Unable to co-ordinate respiration and speech.

\section{References}

[1] H. Ackermann and W. Zeigler, Articulatory deficits in Parkinsonian dysarthria: An acoustic analysis, Journal of Neurology, Neurosurgery and Psychiatry 54 (1996), 1093-1098.

[2] N.P. Connor, J.H. Abbs, K.J. Cole and V.L. Gracco, Parkinsonian deficits in serial multiarticulate movements for speech, Brain 112 (1989), 997-1009.

[3] L. Hartelius and P. Svensson, Speech and swallowing symptoms associated with Parkinson's disease and multiple sclerosis: A survey, Folia Phoniatrica 46 (1994), 9-17.

[4] A.J. Hughes, S.E. Daniel, S. Blankson and A.J. Lees, A clinicopathologic study of 100 cases of Parkinson's disease, Archives of Neurology 50 (1993), 140.

[5] R. Iansek, J.L. Bradshaw, J.G. Phillips, R. Cunnington and M.E. Morris, Interaction of the basal ganglia and supplementary motor area in the elaboration of movement, in: Motor control and sensory integration: Issues and directions, D.J. Glencross and J.P. Piek, eds, Elsevier, New York, 1995, pp. 37-59.
[6] J.A. Logemann, H.B. Fisher, B. Boshes and E.B. Blonsky, Frequency and cooccurrence of vocal tract dysfunctions in the speech of a large sample of parkinson patients, Journal of Speech and Hearing Disorders 43 (1978), 47-57.

[7] C. Marigliani, D. Jacks, S. Gates, S. Wilson, R. Iansek, and T. Matyas, The Functional Parkinson's Communication Profile (unpublished manuscript).

[8] E.J. Metter and W.R. Hanson, Clinical and acoustic variability in hypokinetic dysarthria, Journal of Communication Disorders 19 (1986), 347-366.

[9] M.E. Morris, R. Iansek, T.A. Matyas and J.J. Summers, The pathogenesis of gait hypokinesia in Parkinson's disease, Brain 117 (1994), 1169-1181.

[10] M.E. Morris, R. Iansek, T.A. Matyas, and J.J. Summers, Stride length regulation in Parkinson's disease: Normalising strategies and underlying mechanisms, Brain 119 (1996), 551-568.

[11] W.J. Mutch, I. Dingwall-Fordyce, A.W. Downie, J.G. Patterson and S.K. Roy, Parkinson's disease in a Scottish city, British Medical Journal 293 (1986), 675-677.

[12] D.J. Ostry, Velocity curves of human arm and speech movements, Experimental Brain Research 68 (1987), 37-46.

[13] D.J. Ostry, E. Keller and A. Parush, Similarities in the control of the speech articulators and the limb: Kinematics of tongue dorsum movement in speech, Journal of Experimental Psychology: Human Perception and Performance 9 (1983), 622-623.

[14] G. Selby, Parkinson's disease, in: Handbook of Clinical Neurology, Vol. 6, P.J. Vinken and G.W. Bruyn, eds, North Holland, 1968, pp. 173-211.

[15] W.S. Torgerson, Theory and methods of scaling, John Wiley \& Sons, New York, 1965.

[16] J. Volkmann, J.V.H. Hefter, H.W. Lange and H.J. Freund, Impairment of temporal organisation of speech in basal ganglia diseases, Brain and Language 43 (1992), 386-399.

[17] D.D. Webster, Critical analysis of the disability in Parkinson's disease, Modern Treatment 5 (1968), 257-282. 


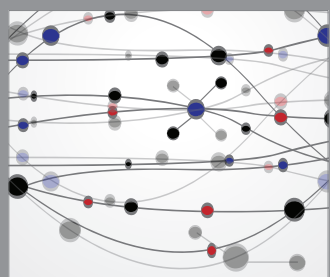

The Scientific World Journal
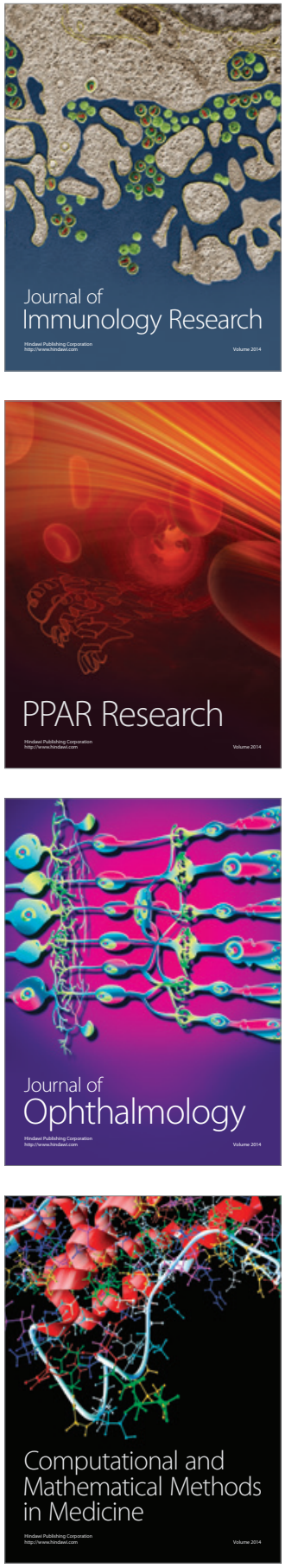

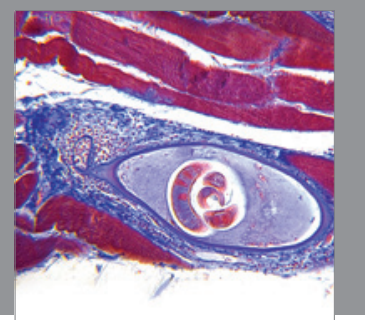

Gastroenterology

Research and Practice
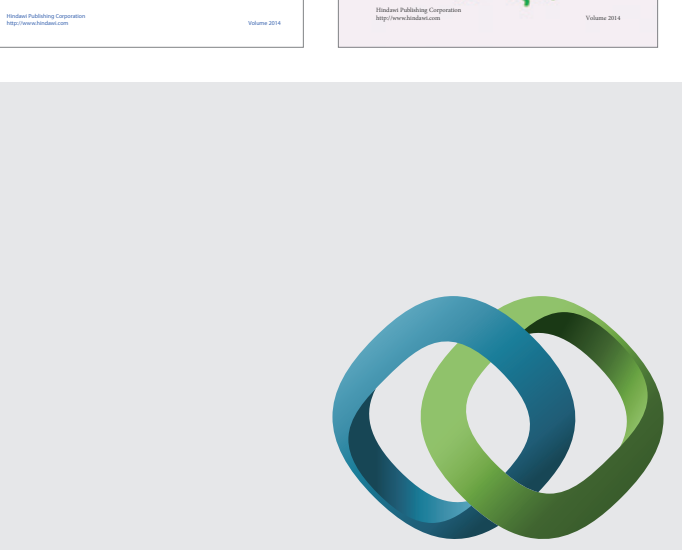

\section{Hindawi}

Submit your manuscripts at

http://www.hindawi.com
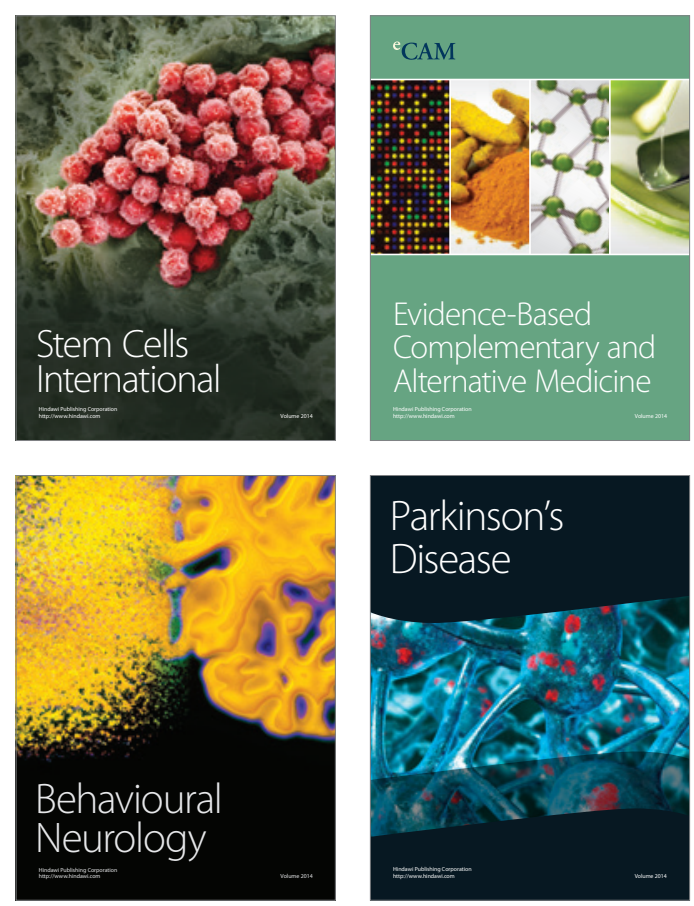

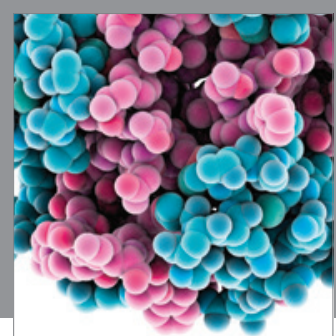

Journal of
Diabetes Research

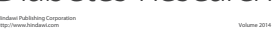

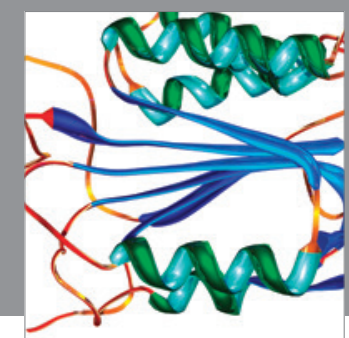

Disease Markers
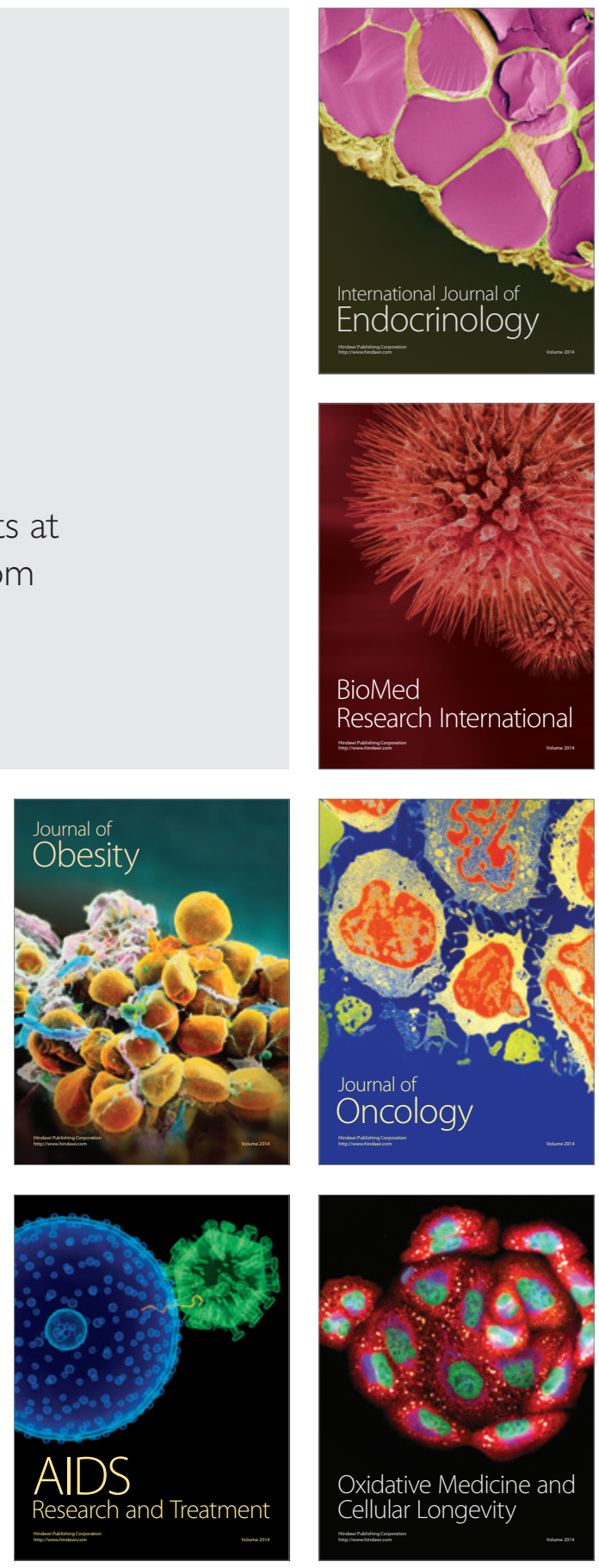